\title{
PAPER
}

Cite this: New J. Chem., 2014, 38, 809

\section{Synthesis and DPPH radical scavenging activity of novel compounds obtained from tyrosol and cinnamic acid derivatives}

\author{
Maurizio Barontini, ${ }^{a}$ Roberta Bernini, ${ }^{* a}$ Isabella Carastro, ${ }^{a}$ Patrizia Gentili ${ }^{b}$ and \\ Annalisa Romani ${ }^{\complement}$
}

Tyrosol, a naturally occurring phenolic compound poorly attractive as an antioxidant because of its weak efficacy, was used as starting material to obtain novel compounds. The synthesis is based on a trifluoroacetic acid-mediated hydroarylation of cinnamic esters with tyrosol to produce 4-aryl-3,4-dihydrocoumarins, molecules of biological interest, followed by a basic hydrolysis to give the corresponding ring opening products. Unreported mechanistic investigations confirmed that the first step resulted from an electrophilic aromatic substitution and an intramolecular transesterification. Final products exhibited DPPH radical scavenging activity significantly higher than tyrosol.
Received (in Montpellier, France) 29th September 2013, Accepted 2nd December 2013

DOI: 10.1039/c3nj01180a

www.rsc.org/njc acid 5 (caffeic acid) and 4-hydroxy-3,5-dimethoxycinnamic acid 6 (sinapic acid), responsible for the beneficial health effects associated with cereal consumption (Fig. 1). ${ }^{11}$ In contrast, 2-(4-hydroxyphenyl)ethanol 2 (tyrosol) shows a weak anti-oxidative efficacy. ${ }^{12}$ Despite this property, in the last few years tyrosol has attracted the attention of organic chemists and pharmacologists as a versatile substrate for the synthesis of a variety of esters exhibiting diverse biological effects including antioxidant, anti-cancer, antimicrobial and antileishmania activities. ${ }^{13}$ In this context, in our laboratory we utilized tyrosol for the preparation of biologically and industrially-relevant catechols that showed antioxidant and antiproliferative effects on human colon cancer cells. ${ }^{14}$ Continuing this research, we describe here the synthesis of novel DPPH (2,2-diphenyl-1-picrylhydrazyl) radical scavengers. As shown in Scheme 1, the key step of our strategy is the preparation of 4-aryl-3,4-dihydrocoumarins. In the literature<smiles>OCCc1ccc(O)c(O)c1</smiles>

1<smiles>O=C(O)/C=C/c1ccc(O)cc1</smiles>

3<smiles>O=C(O)/C=C/c1ccc(O)c(O)c1</smiles>

5<smiles>OCCc1ccc(O)cc1</smiles>

2<smiles>COc1cc(/C=C/C(=O)O)ccc1O</smiles><smiles>COc1cc(/C=C/C(=O)O)cc(OC)c1O</smiles>

6
Fig. 1 Representative naturally occurring phenolic compounds. 
<smiles>[R]c1cc(C(CC(=O)O)c2cc(CCO)ccc2O)cc([R])c1[R]</smiles><smiles>[R20]CCc1ccc2c(c1)[C@H](c1cc([R3])c([R])c([R])c1)CC(=O)O2</smiles>

Target compounds

4-Aryl-3,4dihydrocoumarin derivatives<smiles>C=C</smiles><smiles>[R]OCCc1ccc(O)cc1</smiles>

Scheme 1 Synthetic strategy to obtain novel tyrosol derivatives.

several synthetic methods have been described for the synthesis of this class of compounds including catalytic hydrogenation of coumarins, ${ }^{15}$ reaction of alkenyl carbene chromium(0) complexes with ketene acetals, ${ }^{16}$ reaction of Meldrum's acid or 5-alkylidene Meldrum's acid with phenols, ${ }^{17}$ rhodium-mediated reaction of 3-(2-hydroxyphenyl)-cyclobutanones, ${ }^{18}$ Lewis acid catalyzed reaction of acrylonitrile with phenols, ${ }^{19}$ hydroarylation of cinnamic acid derivatives with alkyl phenols under acidic conditions ${ }^{20}$ or microwave irradiation. ${ }^{21}$ Among them, hydroarylation reaction seems to be of interest allowing the formation of C-C bonds with high atom economy from simple phenol substrates. ${ }^{22}$ A mild and convenient version is the trifluoroacetic acid-mediated hydroarylation. $^{23}$ On the basis of these literature data, we firstly explored the potentiality of this procedure using tyrosol and cinnamic acid derivatives as starting materials in order to obtain novel 4-aryl-3,4-dihydrocoumarins, precursors of our target compounds.

\section{Results and discussion}

Firstly, we selectively protected the alcoholic functionality of tyrosol 2, the carboxylic and phenolic moieties of cinnamic acids 3-6. Thus, tyrosol was converted into the corresponding tyrosol methyl carbonate 7 by an efficient and simple procedure using dimethyl carbonate (DMC) in the presence of 1,8-diazabicyclo[5.4.0]undec-7-ene (DBU), as shown in Scheme 2. ${ }^{24}$

Cinnamic acids 3-6 were converted into the corresponding methyl cinnamates $\mathbf{8 - 1 0}$ by reaction with potassium carbonate and an excess of dimethyl sulfate. The yields of the final products were comparable to those obtained by the Wittig reactions between the corresponding benzaldehydes and the appropriate phosphorane derivatives. ${ }^{25-27}$ Finally, cinnamic acids 3-6 were converted into $p$-acetoxy methyl cinnamates $\mathbf{1 5 - 1 8}$ by reaction of<smiles>COC(=O)OCCc1ccc(O)cc1</smiles>

Scheme 2 Carboxymethylation of tyrosol 1 with DMC/DBU.<smiles>[R]c1cc(/C=C/C(=O)O)cc([R2])c1O</smiles>

3: $\mathrm{R}_{1}=\mathrm{R}_{2}=\mathrm{H}$

4: $\mathrm{R}_{1}=\mathrm{H} ; \mathrm{R}_{2}=\mathrm{OCH}_{3}$

5: $\mathrm{R}_{1}=\mathrm{H} ; \mathrm{R}_{2}=\mathrm{OH}$

6: $\mathrm{R}_{1}=\mathrm{R}_{2}=\mathrm{OCH}_{3}$

b)<smiles>[R]c1cc(/C=C/C(=O)O[Na])cc([R2])c1O[CH]</smiles>

11: $\mathrm{R}_{1}=\mathrm{R}_{2}=\mathrm{H}, 95 \%$

12: $\mathrm{R}_{1}=\mathrm{H} ; \mathrm{R}_{2}=\mathrm{OCH}_{3}, 90 \%$

13: $\mathrm{R}_{1}=\mathrm{H} ; \mathrm{R}_{2}=\mathrm{OAc}, 92 \%$

14: $\mathrm{R}_{1}=\mathrm{R}_{2}=\mathrm{OCH}_{3}, 90 \%$

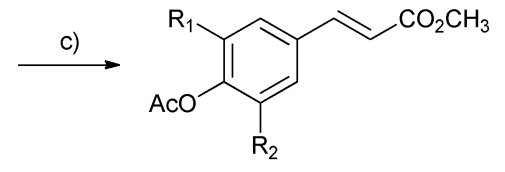

15: $\mathrm{R}_{1}=\mathrm{R}_{2}=\mathrm{H}, 92 \%$

16: $\mathrm{R}_{1}=\mathrm{H} ; \mathrm{R}_{2}=\mathrm{OCH}_{3}, 90 \%$

17: $\mathrm{R}_{1}=\mathrm{H} ; \mathrm{R}_{2}=\mathrm{OAC}, 88 \%$

18: $\mathrm{R}_{1}=\mathrm{R}_{2}=\mathrm{OCH}_{3}, 92 \%$
a) $\left(\mathrm{CH}_{3}\right)_{2} \mathrm{SO}_{4} / \mathrm{K}_{2} \mathrm{CO}_{3}$, acetone, rt, $24 \mathrm{~h}$
b) $\mathrm{Ac}_{2} \mathrm{O}$, dry Py, rt, $24 \mathrm{~h}$
c) $\left(\mathrm{CH}_{3}\right)_{2} \mathrm{SO}_{4} / \mathrm{K}_{2} \mathrm{CO}_{3}$, acetone, $\mathrm{rt}, 8 \mathrm{~h}$

Scheme 3 Methylation and acetylation reactions of cinnamic acids 3-6.

the phenolic moiety with acetic anhydride in dry pyridine followed by methylation of the carboxylic group with potassium carbonate and dimethyl sulfate (Scheme 3).<smiles>COC(=O)OCCc1ccc(O)cc1</smiles>

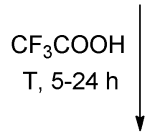<smiles>[R]c1cc(/C=C/C(C)=O)cc([R])c1[R]</smiles>

8: $\mathrm{R}_{1}=\mathrm{R}_{2}=\mathrm{H} ; \mathrm{R}_{3}=\mathrm{OCH}_{3}$ 9: $\mathrm{R}_{1}=\mathrm{H} ; \mathrm{R}_{2}=\mathrm{R}_{3}=\mathrm{OCH}_{3}$ 10: $\mathrm{R}_{1}=\mathrm{R}_{2}=\mathrm{R}_{3}=\mathrm{OCH}_{3}$ 15: $\mathrm{R}_{1}=\mathrm{R}_{2}=\mathrm{H} ; \mathrm{R}_{3}=\mathrm{OAC}$ 16: $\mathrm{R}_{1}=\mathrm{H} ; \mathrm{R}_{2}=\mathrm{OCH}_{3} ; \mathrm{R}_{3}=\mathrm{OAC}$ 17: $\mathrm{R}_{1}=\mathrm{H} ; \mathrm{R}_{2}=\mathrm{R}_{3}=\mathrm{OAC}$ 18: $\mathrm{R}_{1}=\mathrm{R}_{2}=\mathrm{OCH}_{3} ; \mathrm{R}_{3}=\mathrm{OAC}$<smiles>[R]c1cc(C2CC(=O)Oc3ccc(CCOC(=O)OC)cc32)cc([R])c1[R]</smiles>

19: $\mathrm{R}_{1}=\mathrm{R}_{2}=\mathrm{H} ; \mathrm{R}_{3}=\mathrm{OCH}_{3}$ 20: $\mathrm{R}_{1}=\mathrm{H} ; \mathrm{R}_{2}=\mathrm{R}_{3}=\mathrm{OCH}_{3}$ 21: $\mathrm{R}_{1}=\mathrm{R}_{2}=\mathrm{R}_{3}=\mathrm{OCH}_{3}$ 22: $\mathrm{R}_{1}=\mathrm{R}_{2}=\mathrm{H} ; \mathrm{R}_{3}=\mathrm{OH}$ 23: $\mathrm{R}_{1}=\mathrm{H} ; \mathrm{R}_{2}=\mathrm{OCH}_{3} ; \mathrm{R}_{3}=\mathrm{OH}$

24: $\mathrm{R}_{1}=\mathrm{H} ; \mathrm{R}_{2}=\mathrm{R}_{3}=\mathrm{OH}$ 25: $\mathrm{R}_{1}=\mathrm{R}_{2}=\mathrm{OCH}_{3} ; \mathrm{R}_{3}=\mathrm{OH}$
Scheme 4 TFA-mediated reaction of tyrosol methyl carbonate 7 with cinnamic esters 8-10 and 15-18. 
Table 1 Experimental conditions of the reaction depicted in Scheme 4

\begin{tabular}{|c|c|c|c|}
\hline Entry & Cinnamic ester & Experimental conditions ${ }^{a}$ & Product $^{b}$ (yield $\left.\%\right)$ \\
\hline 1 & 8 & $25{ }^{\circ} \mathrm{C}, 24 \mathrm{~h}$ & 19: 76 \\
\hline 2 & 8 & Reflux, $5 \mathrm{~h}$ & 19: 78 \\
\hline 3 & 9 & $25^{\circ} \mathrm{C}, 24 \mathrm{~h}$ & 20: 70 \\
\hline 4 & 9 & Reflux, $5 \mathrm{~h}$ & 20: 74 \\
\hline 5 & 10 & $25{ }^{\circ} \mathrm{C}, 24 \mathrm{~h}$ & 21: 68 \\
\hline 6 & 10 & Reflux, $5 \mathrm{~h}$ & 21: 64 \\
\hline 7 & 15 & $25{ }^{\circ} \mathrm{C}, 24 \mathrm{~h}$ & 22: 42 \\
\hline 8 & 15 & Reflux, $6 \mathrm{~h}$ & 22: 52 \\
\hline 9 & 16 & $25{ }^{\circ} \mathrm{C}, 24 \mathrm{~h}$ & 23: 40 \\
\hline 10 & 16 & Reflux, $6 \mathrm{~h}$ & 23: 50 \\
\hline 11 & 17 & $25{ }^{\circ} \mathrm{C}, 24 \mathrm{~h}$ & 24: 38 \\
\hline 12 & 17 & Reflux, 6 h & 24: 42 \\
\hline 13 & 18 & $25{ }^{\circ} \mathrm{C}, 24 \mathrm{~h}$ & 25: 40 \\
\hline 14 & 18 & Reflux, $5 \mathrm{~h}$ & 25: 44 \\
\hline
\end{tabular}

${ }^{a}$ Tyrosol methyl carbonate $7(0.5 \mathrm{mmol})$; esters $\mathbf{8 - 1 0}$ and $\mathbf{1 5 - 1 8}$ $(0.5 \mathrm{mmol})$; trifluoroacetic acid: $2 \mathrm{~mL} .{ }^{b}$ Calculated after chromatographic purification.

Hydroarylation reactions of cinnamic esters 8-10 and 15-18 with tyrosol methyl carbonate 7 in trifluoroacetic acid are depicted in Scheme 4. Experimental results showed that 4-aryl-3,4-dihydrocoumarins 19-21 derived from cinnamic esters bearing electrondonating groups were obtained in satisfactory yields both at room temperature and reflux temperature (Table 1, entries 1-6); 4-aryl-3,4dihydrocoumarins 22-25 derived from cinnamic esters bearing an electron-withdrawing group at the para-position (an acetoxy group) were also isolated in lower yields at reflux temperature (Table 1, entries 7-14).

In the literature the hypothesized mechanism of the TFAhydroarylation reaction, suggested on the basis of the electronic substituent effects on cinnamic ester derivatives, consists of the aromatic electrophilic substitution by the protonated cinnamic ester on the phenolic substrate, followed by the intramolecular transesterification to afford the dihydrocoumarin. ${ }^{20}$ In order to confirm this hypothesis, we carried out the reactions of ester 8 in combination with 2,6-dimethylphenol 26 and 2,4-dimethylphenol 27 in trifluoroacetic acid (Scheme 5). According to the proposed mechanism, phenol 26, exhibiting two methyl groups in both the ortho-positions and the free para-position, gave 28 as the only product; in contrast, phenol 27 , showing one free orthoposition, produced the dihydrocoumarin 29 . Both at $25{ }^{\circ} \mathrm{C}$ and reflux temperature, compounds $\mathbf{2 8}$ and $\mathbf{2 9}$ were obtained as the only reaction products.

Novel 4-aryl-3,4-dihydrocoumarins 19-25 appear to be interesting molecules from the biological point of view. As a matter of fact, several compounds of these class have been shown to possess many biological properties such as antiherpetic, ${ }^{25}$ estrogenic, ${ }^{26}$ antimicrobial, ${ }^{27}$ anti-inflammatory, ${ }^{28}$ cytotoxic, ${ }^{29}$ and antifungal activities. $^{30}$ In addition, many dihydrocoumarins are used as synthetic intermediates of pharmaceuticals and flavoring agents of foods such as drinks, yogurt, and cakes. ${ }^{31}$

Finally, we carried out the basic hydrolysis of compounds 19-25. Under these conditions both the opening of the lactonic ring and deprotection of the carbonate moiety of the tyrosol skeleton were observed to produce tyrosol derivatives 30, 31, 32, 33, 34 and 36 (Scheme 6). Unfortunately, we were not able to isolate a pure sample of tyrosol derivative 35 , probably due to its high polarity.

Compounds 30, 31, 32, 33, 34 and 36 were evaluated for their radical scavenging capacity by using the DPPH radical test assay. ${ }^{32}$ The antioxidant activity was defined as the amount of compound necessary to decrease the initial DPPH concentration by $50 \%$ and expressed as $\mathrm{EC}_{50}$ (efficient concentration $=$ mmol tyrosol derivative/mmol DPPH). As shown in Table 2, all novel products

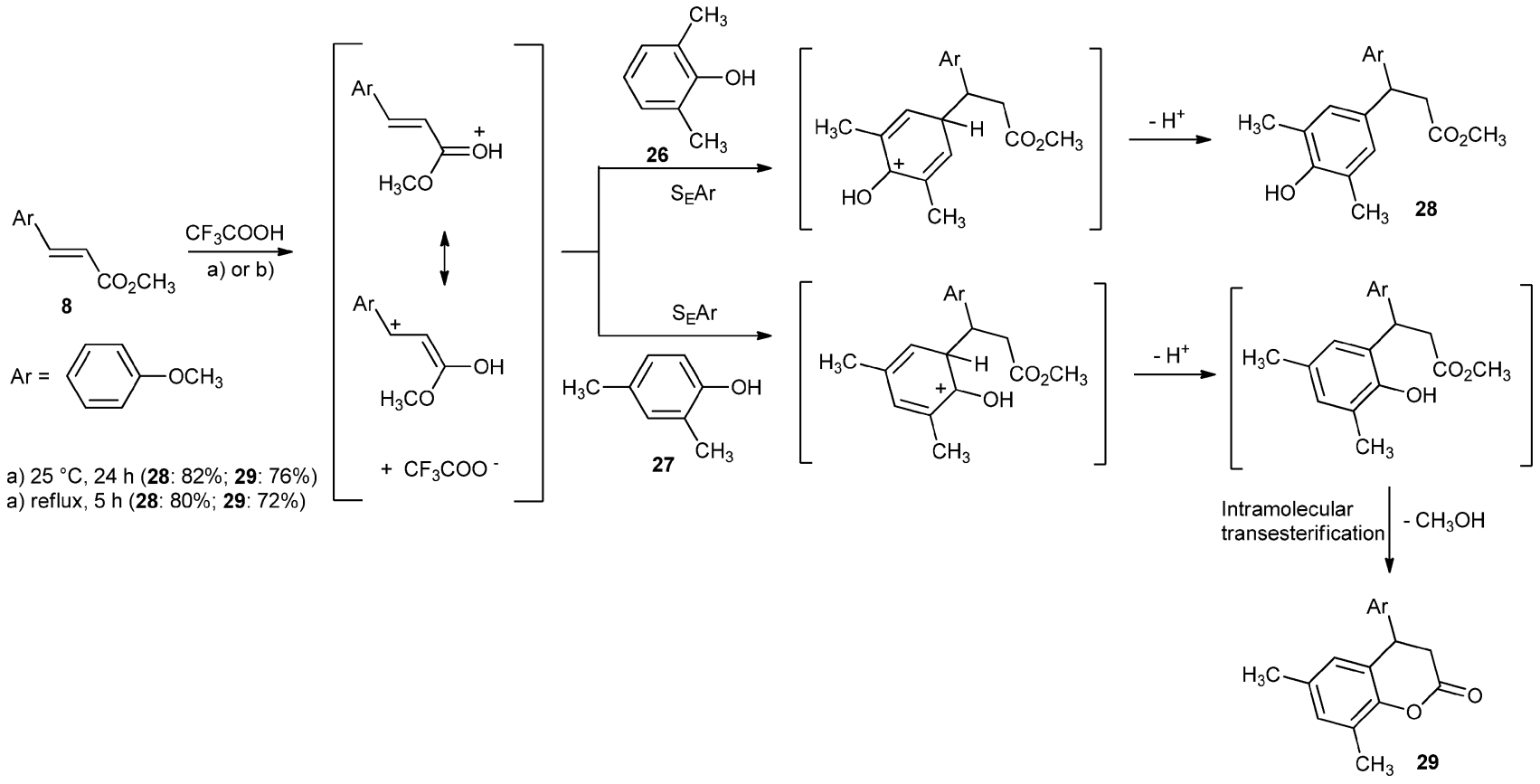

Scheme 5 Mechanistic investigations with cinnamic ester $\mathbf{8}$ and phenols $\mathbf{2 6}$ and 27. 
<smiles>[R]c1cc(C2CC(=O)Oc3ccc(CCOC(=O)OC)cc32)cc([R2])c1[R3]</smiles>

$19-25$

19: $\mathrm{R}_{1}=\mathrm{R}_{2}=\mathrm{H} ; \mathrm{R}_{3}=\mathrm{OCH}_{3}$ 20: $\mathrm{R}_{1}=\mathrm{H} ; \mathrm{R}_{2}=\mathrm{R}_{3}=\mathrm{OCH}_{3}$ 21: $\mathrm{R}_{1}=\mathrm{R}_{2}=\mathrm{R}_{3}=\mathrm{OCH}_{3}$

22: $\mathrm{R}_{1}=\mathrm{R}_{2}=\mathrm{H} ; \mathrm{R}_{3}=\mathrm{OH}$

23: $\mathrm{R}_{1}=\mathrm{H} ; \mathrm{R}_{2}=\mathrm{OCH}_{3} ; \mathrm{R}_{3}=\mathrm{OH}$

24: $\mathrm{R}_{1}=\mathrm{H} ; \mathrm{R}_{2}=\mathrm{R}_{3}=\mathrm{OH}$

25: $\mathrm{R}_{1}=\mathrm{R}_{2}=\mathrm{OCH}_{3} ; \mathrm{R}_{3}=\mathrm{OH}$<smiles>[R]c1cc(C(CC(=O)O)c2cc(CCO)ccc2O)cc([R])c1[123I]</smiles>

30: $\mathrm{R}_{1}=\mathrm{R}_{2}=\mathrm{H} ; \mathrm{R}_{3}=\mathrm{OCH}_{3}(90 \%)$

31: $\mathrm{R}_{1}=\mathrm{H} ; \mathrm{R}_{2}=\mathrm{R}_{3}=\mathrm{OCH}_{3}(92 \%)$

32: $\mathrm{R}_{1}=\mathrm{R}_{2}=\mathrm{R}_{3}=\mathrm{OCH}_{3}(92 \%)$

33: $\mathrm{R}_{1}=\mathrm{R}_{2}=\mathrm{H} ; \mathrm{R}_{3}=\mathrm{OH}(88 \%)$

34: $\mathrm{R}_{1}=\mathrm{H} ; \mathrm{R}_{2}=\mathrm{OCH}_{3} ; \mathrm{R}_{3}=\mathrm{OH}(90 \%)$

35: $\mathrm{R}_{1}=\mathrm{H} ; \mathrm{R}_{2}=\mathrm{R}_{3}=\mathrm{OH}$ (traces)

36: $\mathrm{R}_{1}=\mathrm{R}_{2}=\mathrm{OCH}_{3} ; \mathrm{R}_{3}=\mathrm{OH}(92 \%)$

Scheme 6 Basic hydrolysis of 4-aryl-3,4-dihydrocoumarins 19-25.

Table 2 DPPH radical-scavenging effect of tyrosol 2 and compounds 30-34 and 36

Compound<smiles>OCCc1ccc(O)cc1</smiles>

$\mathrm{EC}_{50}{ }^{a}$

$21 \pm 1.0$

Compound

$\mathrm{EC}_{50}{ }^{a}$

${ }^{a} \mathrm{Mmol}$ isochroman/mmol DPPH radical.

showed a significant radical-scavenging activity. Among them, the most active was compound 36; as a general trend, the substitution of a methoxy group with an hydroxyl group in the acidic frame produced an increase of activity (compare compound 30 with 33; 31 with 34; and 32 with 36) as already reported in the literature and also observed by us. ${ }^{14}$

\section{Conclusions}

Tyrosol, a naturally occurring phenolic compound poorly attractive as an antioxidant because of its weak efficacy, was used as starting material for the preparation of novel bioactive compounds by a twostep procedure: (1) a trifluoroacetic acid-mediated hydroarylation of cinnamic esters and (2) a basic hydrolysis of the corresponding 4-aryl-3,4-dihydrocoumarins. Unreported mechanistic investigations confirmed that the hydroarylation process proceeded by an electrophilic substitution followed by an intramolecular esterification. Pure samples of final compounds were evaluated for the DPPH radical scavenging activity. Experimental results demonstrated that all compounds showed an effect significantly higher than tyrosol and their efficacy increased with the presence of one hydroxyl group in the aromatic ring of the acidic frame.

\section{Experimental section}

\section{Materials and methods}

Reagents and solvents were supplied by Sigma Aldrich (Milan, Italy) and used without further purification. Tyrosol methyl carbonate 7 was prepared according to the procedure already reported by us. ${ }^{24}$ Silica gel 60 F254 plates and silica gel 60 were obtained from Merck (Milan, Italy). ${ }^{1} \mathrm{H}$ NMR and ${ }^{13} \mathrm{C}$ NMR spectra were recorded on a Bruker $200 \mathrm{MHz}$ spectrometer using $\mathrm{CDCl}_{3}$ and $\mathrm{CD}_{3} \mathrm{OD}$ as solvents. Chemical shifts are expressed in parts per million ( $\delta$ scale) and coupling constants in Hertz. GC-MS analyses were performed on a Shimadzu VG 70/250S apparatus equipped with a Supelco SLB ${ }^{\mathrm{TM}}$ $5 \mathrm{~ms}$ column (30 m, $0.25 \mathrm{~mm}$ and $0.25 \mu \mathrm{m}$ film thickness). 
The analyses were performed using an isothermal temperature profile of $100{ }^{\circ} \mathrm{C}$ for $2 \mathrm{~min}$, followed by a $10{ }^{\circ} \mathrm{C} \mathrm{min}{ }^{-1}$ temperature gradient until $280{ }^{\circ} \mathrm{C}$ for $15 \mathrm{~min}$. The injector temperature was $280{ }^{\circ} \mathrm{C}$. High Resolution Mass Spectrometry (HRMS) analyses were performed using a Micromass Q-TOF micro Mass Spectrometer (Waters).

\section{Synthesis}

Methylation of cinnamic acids (3)-(6). Cinnamic acid 3, 4 or 6 $(1.0 \mathrm{mmol})$ was solubilized in acetone $(5 \mathrm{~mL})$ at room temperature; then, potassium carbonate $(2.0 \mathrm{mmol})$ and dimethyl sulfate $(2.0 \mathrm{mmol})$ were added. The mixture was kept under magnetic stirring at room temperature for $24 \mathrm{~h}$. After the work-up, the final product $(8,9$ or 10) was purified on a silica gel chromatographic column using hexane-ethyl acetate $=9 / 1$ as an eluent.

(E)-Methyl 3-(4-methoxyphenyl)acrylate (8). Yield: $92 \%$; colorless oil; spectroscopic data are according to the literature. ${ }^{33}$

(E)-Methyl 3-(3,4-dimethoxyphenyl)acrylate (9). Yield: 90\%; colorless oil; spectroscopic data are according to the literature. ${ }^{34}$

(E)-Methyl 3-(3,4,5-trimethoxyphenyl)acrylate (10). Yield: 88\%; colorless oil; spectroscopic data are according to the literature. ${ }^{35}$

Acetylation of cinnamic acids (3)-(6). To a solution of cinnamic acid 3, 4,5 or $6(1.0 \mathrm{mmol})$ in dry pyridine $(1.5 \mathrm{~mL})$ was added acetic anhydride $(1.5 \mathrm{~mL})$. The mixture was stirred at room temperature overnight. Then, the reaction mixture was poured into ice-water $(5 \mathrm{~mL})$ and treated with $3 \mathrm{M} \mathrm{HCl}$. The precipitated product was filtered and washed with water and diethyl ether. Pure samples of compounds $(\mathbf{1 1}, \mathbf{1 2}, \mathbf{1 3}$, and 14) were obtained after purification in a silica gel chromatographic column using hexane-ethyl acetate $=8 / 2$ as an eluent.

(E)-3-(4-Acetoxyphenyl)acrylic acid (11). Yield: 95\%; colorless oil; spectroscopic data are according to the literature. ${ }^{36}$

(E)-3-(4-Acetoxy-3-methoxyphenyl)acrylic acid (12). Yield: 90\%; colorless oil; spectroscopic data are according to the literature. ${ }^{37}$

(E)-3-(3,4-Diacetoxyphenyl)acrylic acid (13). Yield: 92\%; colorless oil; spectroscopic data are according to the literature. ${ }^{38}$

(E)-3-(4-Acetoxy-3,5-dimethoxyphenyl)acrylic acid (14). Yield: 90\%; colorless oil; spectroscopic data are according to the literature. ${ }^{37}$

Methylation of cinnamic acid derivatives (11)-(14). Cinnamic acid $11,12,13$ or $14(1.0 \mathrm{mmol})$ was solubilized in acetone $(5 \mathrm{~mL})$ at room temperature. Then potassium carbonate $(1.0 \mathrm{mmol})$ and dimethyl sulfate $(1.0 \mathrm{mmol})$ were added and the mixture was kept under magnetic stirring at room temperature for $8 \mathrm{~h}$. After the work-up, the final product $(\mathbf{1 1}, \mathbf{1 2}, \mathbf{1 3}$, or 14) was purified in a silica gel chromatographic column using hexane-ethyl acetate = $8 / 2$ as an eluent.

(E)-Methyl 3-(4-acetoxyphenyl)acrylate (15). Yield: 92\%; colorless oil; spectroscopic data are according to the literature. ${ }^{39}$

(E)-Methyl 3-(4-acetoxy-3-methoxyphenyl)acrylate (16). Yield: 90\%; colorless oil; ${ }^{1} \mathrm{H}$ NMR $\left(\mathrm{CDCl}_{3}, 200 \mathrm{MHz}\right) \delta: 7.66(\mathrm{~d}, J=$ $16.0 \mathrm{~Hz}, 1 \mathrm{H}, \mathrm{CH}=\mathrm{CH}), 6.96-7.09(\mathrm{~m}, 3 \mathrm{H}, \mathrm{Ph}-\mathrm{H}), 6.34(\mathrm{~d}, J=$ $16.0 \mathrm{~Hz}, 1 \mathrm{H}, \mathrm{CH}=\mathrm{CH}), 3.84\left(\mathrm{~s}, 3 \mathrm{H}, \mathrm{OCH}_{3}\right), 3.77(\mathrm{~s}, 3 \mathrm{H}$, $\left.\mathrm{CO}_{2} \mathrm{CH}_{3}\right), 2.29\left(\mathrm{~s}, 3 \mathrm{H}, \mathrm{OCOCH}_{3}\right) ;{ }^{13} \mathrm{C} \mathrm{NMR}\left(50 \mathrm{MHz}, \mathrm{CDCl}_{3}\right) \delta$ : $168.7,167.2$, 151.4, 144.1, 141.4, 133.2, 123.2, 121.1, 118.0, 111.3, 55.8, 51.7, 20.6; GC-MS: $250\left(\mathrm{M}^{+}\right), 208,177,145$. $\mathrm{C}_{13} \mathrm{H}_{14} \mathrm{O}_{5}$ requires C, 62.39; H, 5.64; found: C, 62.45; H, 5.60.
(E)-Methyl 3-(3,4-diacetoxyphenyl)acrylate (17). Yield 88\%; colorless oil; ${ }^{1} \mathrm{H}$ NMR $\left(\mathrm{CDCl}_{3}, 200 \mathrm{MHz}\right) \delta: 7.57(\mathrm{~d}, J=16.0 \mathrm{~Hz}$, $1 \mathrm{H}, \mathrm{CH}=\mathrm{CH}), 7.14-7.37(\mathrm{~m}, 3 \mathrm{H}, \mathrm{Ph}-\mathrm{H}), 6.33(\mathrm{~d}, J=16.0 \mathrm{~Hz}, 1 \mathrm{H}$, $\mathrm{CH}=\mathrm{CH}), 3.74\left(3 \mathrm{H}, \mathrm{s}, \mathrm{CO}_{2} \mathrm{CH}_{3}\right), 2.25\left(6 \mathrm{H}, \mathrm{s}, 2 \mathrm{OCOCH}_{3}\right)$; ${ }^{13} \mathrm{C}$ NMR (50 MHz, $\left.\mathrm{CDCl}_{3}\right) \delta: 168.0$ (2C), 166.9, 143.5, 142.8, 142.4, 133.2, 126.3, 123.9, 122.6, 118.9, 51.7, 20.5 (2C); GC-MS: $278\left(\mathrm{M}^{+}\right), 236,194,163,134 . \mathrm{C}_{14} \mathrm{H}_{14} \mathrm{O}_{6}$ requires $\mathrm{C}, 60.43 ; \mathrm{H}$, 5.07; found C, 60.54; H, 5.10 .

(E)-Methyl 3-(4-acetoxy-3,5-dimethoxyphenyl)acrylate (18). Yield 92\%; colorless oil; ${ }^{1} \mathrm{H}$ NMR $\left(\mathrm{CDCl}_{3}, 200 \mathrm{MHz}\right) \delta: 7.54$ $(\mathrm{d}, J=16.0 \mathrm{~Hz}, 1 \mathrm{H}, \mathrm{CH}=\mathrm{CH}), 6.70(\mathrm{~s}, 2 \mathrm{H}, \mathrm{Ph}-\mathrm{H}), 6.32$ $(\mathrm{d}, J=16.0 \mathrm{~Hz}, 1 \mathrm{H}, \mathrm{CH}=\mathrm{CH}), 3.76\left(\mathrm{~s}, 6 \mathrm{H}, 2 \mathrm{OCH}_{3}\right), 3.74$ (s, $\left.3 \mathrm{H}, \mathrm{CO}_{2} \mathrm{CH}_{3}\right), 2.23\left(\mathrm{~s}, 3 \mathrm{H}, \mathrm{OCOCH}_{3}\right) ;{ }^{13} \mathrm{C} \mathrm{NMR}\left(50 \mathrm{MHz}, \mathrm{CDCl}_{3}\right)$ $\delta: 168.4,167.1,152.4(2 \mathrm{C}), 144.5,132.6,130.4,118.0,104.6$ (2C), 56.1 (2C), 51.6, 20.3; GC-MS: $280\left(\mathrm{M}^{+}\right), 238,207,175,163,147,135$, 119. $\mathrm{C}_{14} \mathrm{H}_{16} \mathrm{O}_{6}$ requires $\mathrm{C}, 59.99 ; \mathrm{H}, 5.75$; found $\mathrm{C}, 60.19 ; \mathrm{H} \mathrm{5.70.}$

Reaction of tyrosol methyl carbonate (7) with cinnamates (8)-(10) or (15)-(18). Tyrosol methyl carbonate $7(0.3 \mathrm{mmol})$ and the appropriate cinnamate derivative $8,9,10,15,16,17$ or 18 $(0.5 \mathrm{mmol})$ were kept in trifluoroacetic acid $(2.5 \mathrm{~mL})$ under magnetic stirring at room or reflux temperature for 5-24 h depending on the experiment. In the end, the crude was neutralized with aqueous $\mathrm{NaHCO}_{3}$ and extracted with ethyl acetate. Organic phases were washed with a saturated $\mathrm{NaCl}$ solution and dried on anhydrous $\mathrm{Na}_{2} \mathrm{SO}_{4}$. After evaporation of the solvent, the final product $(19,20,21,22,23,24$, or 25) was purified in a silica gel chromatographic column using hexane-ethyl acetate (8/2 or $7 / 3)$ as an eluent depending on the substrate.

2-[4-(4-Methoxyphenyl)-2-oxochroman-6-yl] ethyl methyl carbonate (19). Yield 76 and $78 \%$; colorless oil; ${ }^{1} \mathrm{H}$ NMR $\left(\mathrm{CDCl}_{3}\right.$, $200 \mathrm{MHz}) \delta: 7.02-7.16$ (4H, m, Ph-H), 6.72-6.87 (m, 3H, Ph-H), 4.20-4.27 (m, $3 \mathrm{H}, \mathrm{CH}_{2}$ and $\left.\mathrm{CH}\right), 3.78\left(\mathrm{~s}, 3 \mathrm{H}, \mathrm{OCH}_{3}\right), 3.72(\mathrm{~s}, 3 \mathrm{H}$, $\left.\mathrm{CO}_{2} \mathrm{CH}_{3}\right), 2.87-3.00\left(\mathrm{~m}, 2 \mathrm{H}, \mathrm{CH}_{2}\right), 2.86\left(\mathrm{t}, J=6.7 \mathrm{~Hz}, 2 \mathrm{H}, \mathrm{CH}_{2}\right)$; ${ }^{13} \mathrm{C}$ NMR $\left(50 \mathrm{MHz}, \mathrm{CDCl}_{3}\right) \delta: 167.7,159.0,155.6,150.5$, 133.8, 132.1, 130.1, 129.1, 128.7, 128.6, 126.2, 117.2, 115.4, 114.5, 68.1, 55.3, 54.7, 39.9, 37.2, 34.4; GC-MS: $356\left(\mathrm{M}^{+}\right), 280$, 262, 237, 207. $\mathrm{C}_{20} \mathrm{H}_{20} \mathrm{O}_{6}$ requires C, 67.41; H, 5.66; found C, $67.50 ; \mathrm{H}, 5.60$.

2-[4-(3,4-Dimethoxyphenyl)-2-oxochroman-6-yl]ethyl methyl carbonate (20). Yield 70 and $74 \%$; colorless oil; ${ }^{1} \mathrm{H} \mathrm{NMR}\left(\mathrm{CDCl}_{3}\right.$, $200 \mathrm{MHz}) \delta$ : 7.02-7.16 (m, 2H, Ph-H); 6.63-6.83 (m, 4H, Ph-H), 4.20-4.27 (m, $3 \mathrm{H}, \mathrm{CH}_{2}$ and $\left.\mathrm{CH}\right), 3.81\left(3 \mathrm{H}, \mathrm{s}, \mathrm{OCH}_{3}\right), 3.77(3 \mathrm{H}, \mathrm{s}$, $\left.\mathrm{OCH}_{3}\right), 3.71\left(3 \mathrm{H}, \mathrm{s}, \mathrm{CO}_{2} \mathrm{CH}_{3}\right), 2.96-3.01\left(\mathrm{~m}, 2 \mathrm{H}, \mathrm{CH}_{2}\right), 2.88(\mathrm{t}, J=$ $\left.7.1 \mathrm{~Hz}, 2 \mathrm{H}, \mathrm{CH}_{2}\right) ;{ }^{13} \mathrm{C} \mathrm{NMR}\left(50 \mathrm{MHz}, \mathrm{CDCl}_{3}\right) \delta: 167.6,155.6,150.5$, 149.5, 148.6, 133.8, 132.6, 129.2, 128.6, 126.1, 119.7, 117.2, 111.6, 110.5, 68.1, 55.9 (2C), 54.7, 40.4, 37.1, 34.4; GC-MS: $386\left(\mathrm{M}^{+}\right), 310$, 292, 277, 237. $\mathrm{C}_{21} \mathrm{H}_{22} \mathrm{O}_{7}$ requires C, 65.28; H 5.74; found $\mathrm{C}, 65.42$; H, 5.84 .

Methyl [2-(2-oxo-4-(3,4,5-trimethoxyphenyl)chroman)-6-yl-]ethyl carbonate (21). Yield 68 and 64\%; colorless oil; ${ }^{1} \mathrm{H}$ NMR $\left(\mathrm{CDCl}_{3}, 200 \mathrm{MHz}\right) \delta: 6.87-7.17(\mathrm{~m}, 3 \mathrm{H}, \mathrm{Ph}-\mathrm{H}), 6.32(\mathrm{~s}, 2 \mathrm{H}$, $\mathrm{Ph}-\mathrm{H}), 4.19-4.29\left(\mathrm{~m}, 3 \mathrm{H}, \mathrm{CH}_{2}\right.$ and $\left.\mathrm{CH}\right), 3.84\left(\mathrm{~s}, 3 \mathrm{H}, 2 \mathrm{OCH}_{3}\right)$, $3.78\left(\mathrm{~s}, 3 \mathrm{H}, \mathrm{OCH}_{3}\right), 3.71\left(3 \mathrm{H}, \mathrm{s}, \mathrm{CO}_{2} \mathrm{CH}_{3}\right), 2.97-3.03(\mathrm{~m}, 2 \mathrm{H}$, $\left.\mathrm{CH}_{2}\right), 2.81\left(2 \mathrm{H}, \mathrm{t}, J=6.8 \mathrm{~Hz}, \mathrm{CH}_{2}\right) ;{ }^{13} \mathrm{C} \mathrm{NMR}\left(50 \mathrm{MHz}, \mathrm{CDCl}_{3}\right) \delta$ : 167.7, 155.6, 150.5 (2C), 146.9, 145.1, 133.8, 132.0, 129.2, 128.7, 126.2, 120.5, 117.2, 114.9, 109.7, 68.1, 55.9, 54.7, 40.5, 37.2, 
34.4, 29.7; GC-MS: $416\left(\mathrm{M}^{+}\right), 340,322,307,281,267 . \mathrm{C}_{22} \mathrm{H}_{24} \mathrm{O}_{8}$ requires $\mathrm{C}, 63.45 ; \mathrm{H}, 5.81$; found $\mathrm{C} 65.08 ; \mathrm{H}, 5.80$.

2-[4-(4-Hydroxyphenyl)-2-oxochroman-6-yl]ethyl methyl carbonate (22). Yield 42 and $52 \%$; colorless oil; ${ }^{1} \mathrm{H}$ NMR $\left(\mathrm{CDCl}_{3}\right.$, $200 \mathrm{MHz}) \delta$ : 6.93-7.15 (m, 5H, Ph-H), 6.71-6.83 (m, 2H, Ph-H), 4.19-4.27 (m, 3H, $\mathrm{CH}_{2}$ and $\left.\mathrm{CH}\right), 3.72\left(\mathrm{~s}, 3 \mathrm{H}, \mathrm{CO}_{2} \mathrm{CH}_{3}\right), 2.94-2.99$ $\left(\mathrm{m}, 2 \mathrm{H}, \mathrm{CH}_{2}\right), 2.86\left(\mathrm{t}, J=6.9 \mathrm{~Hz}, 2 \mathrm{H}, \mathrm{CH}_{2}\right) ;{ }^{13} \mathrm{C}$ NMR $(50 \mathrm{MHz}$, $\left.\mathrm{CDCl}_{3}\right) \delta: 168.2,155.4,150.4,148.2,133.9,131.9,129.1,128.7$ (2C), 128.5, 126.2, 117.2, 116.0 (2C), 68.1, 54.1, 39.8, 37.2, 34.3; GC-MS: $342\left(\mathrm{M}^{+}\right), 266,248,223,207 . \mathrm{C}_{19} \mathrm{H}_{18} \mathrm{O}_{6}$ requires $\mathrm{C}$, 66.66; H, 5.30; found C, 66.46; H 5.20.

2-[4-(4-Hydroxy-3-methoxyphenyl)-2-oxochroman-6-yl]ethyl methyl carbonate (23). Yield 40 and $50 \%$; colorless oil; ${ }^{1} \mathrm{H}$ NMR $\left(\mathrm{CDCl}_{3}, 200 \mathrm{MHz}\right) \delta: 7.02-7.16(\mathrm{~m}, 2 \mathrm{H}, \mathrm{Ph}-\mathrm{H}) ; 6.83-6.88(\mathrm{~m}, 2 \mathrm{H}$, Ph-H), 6.60-6.65 (m, 2H, Ph-H), 5.60 (s, br, 1H, OH), 4.18-4.27 $\left(\mathrm{m}, 3 \mathrm{H}, \mathrm{CH}_{2}\right.$ and $\left.\mathrm{CH}\right), 3.82\left(\mathrm{~s}, 3 \mathrm{H}, \mathrm{OCH}_{3}\right), 3.72\left(\mathrm{~s}, 3 \mathrm{H}, \mathrm{CO}_{2} \mathrm{CH}_{3}\right)$, 2.98-3.01 (m, 2H, $\left.\mathrm{CH}_{2}\right), 2.87\left(\mathrm{t}, J=6.9 \mathrm{~Hz}, 2 \mathrm{H}, \mathrm{CH}_{2}\right) ;{ }^{13} \mathrm{C} \mathrm{NMR}$ $\left(50 \mathrm{MHz}, \mathrm{CDCl}_{3}\right) \delta: 167.7,155.6,150.4,146.9,145.1,133.8$, 132.0, 129.1, 128.6, 126.1, 120.5, 117.2, 114.8, 109.7, 68.1, 55.9, 54.7, 40.4, 37.2, 34.4. GC-MS: $386\left(\mathrm{M}^{+}\right)$, 372, 296, 278, 253, 223. $\mathrm{C}_{20} \mathrm{H}_{20} \mathrm{O}_{7}$ requires $\mathrm{C}, 64.51 ; \mathrm{H}, 5.41$; found $\mathrm{C}, 64.61 ; \mathrm{H}, 5.31$.

2-[4-(3,4-Dihydroxyphenyl)-2-oxochroman-6-yl]ethyl methyl carbonate (24). Yield 38 and $42 \%$; colorless oil; ${ }^{1} \mathrm{H} \mathrm{NMR}\left(\mathrm{CDCl}_{3}\right.$, $200 \mathrm{MHz}) \delta: 6.78-7.14(\mathrm{~m}, 4 \mathrm{H}, \mathrm{Ph}-\mathrm{H}), 6.57(\mathrm{~d}, J=6.7 \mathrm{~Hz}, 2 \mathrm{H}$, $\mathrm{Ph}-\mathrm{H}), 4.05-4.34\left(\mathrm{~m}, 3 \mathrm{H}, \mathrm{CH}_{2}\right.$ and $\left.\mathrm{CH}\right), 3.72\left(\mathrm{~s}, 3 \mathrm{H}, \mathrm{CO}_{2} \mathrm{CH}_{3}\right)$, 2.85-3.03 (m, 2H, $\left.\mathrm{CH}_{2}\right), 2.87\left(\mathrm{t}, J=6.8 \mathrm{~Hz}, 2 \mathrm{H}, \mathrm{CH}_{2}\right) ;{ }^{13} \mathrm{C} \mathrm{NMR}$ $\left(50 \mathrm{MHz}, \mathrm{CDCl}_{3}\right) \delta: 168.2,155.6,150.4,144.1,143.4,133.8$, 132.7, 128.9, 128.7, 125.9, 119.9, 117.0, 115.6, 114.4, 68.3, 54.9, 39.8, 36.9, 34.4; GC-MS: $358\left(\mathrm{M}^{+}\right)$, 382, 296, 278, 253, 194. $\mathrm{C}_{19} \mathrm{H}_{18} \mathrm{O}_{7}$ requires $\mathrm{C}, 63.68 ; \mathrm{H}, 5.06$; found $\mathrm{C}, 63.88 ; \mathrm{H}, 5.16$.

2-[4-(4-Hydroxy-3,5-dimethoxyphenyl)-2-oxochroman-6-yl] ethyl methyl carbonate (25). Yield 40 and $44 \%$; colorless oil; ${ }^{1} \mathrm{H}$ NMR $\left(\mathrm{CDCl}_{3}, 200 \mathrm{MHz}\right) \delta:$ 6.73-7.02 (m, 3H, Ph-H), 6.34 (s, 2H, Ph-H), 4.17-4.28 (m, $3 \mathrm{H}, \mathrm{CH}_{2}$ and $\left.\mathrm{CH}\right), 3.80\left(6 \mathrm{H}, \mathrm{s}, 2 \mathrm{OCH}_{3}\right), 3.71(3 \mathrm{H}$, $\left.\mathrm{s}, \mathrm{CO}_{2} \mathrm{CH}_{3}\right), 2.90-3.01\left(\mathrm{~m}, 2 \mathrm{H}, \mathrm{CH}_{2}\right), 2.86\left(\mathrm{t}, J=6.8 \mathrm{~Hz}, 2 \mathrm{H}, \mathrm{CH}_{2}\right)$; ${ }^{13} \mathrm{C} \mathrm{NMR}\left(50 \mathrm{MHz}, \mathrm{CDCl}_{3}\right) \delta: 167.6,155.6,150.5,147.4(2 \mathrm{C})$, 134.2, 133.9, 131.2, 130.1, 129.2, 128.7, 126.1, 117.2 (2C), 104.3 (2C), 68.1, 56.3, 54.7, 40.9, 37.1; GC-MS: $402\left(\mathrm{M}^{+}\right), 326,308,293$, 253. $\mathrm{C}_{21} \mathrm{H}_{22} \mathrm{O}_{8}$ requires $\mathrm{C}, 62.68 ; \mathrm{H}, 5.51$; found $\mathrm{C}, 62.48$; H, 5.59.

Methyl 3-(4-hydroxy-3,5-dimethylphenyl)-3-(4-methoxyphenyl)propanoate (28). Yield 80 and $82 \%$; colorless oil; ${ }^{1} \mathrm{H}$ NMR $\left(\mathrm{CDCl}_{3}, 200 \mathrm{MHz}\right) \delta: 7.16-7.19(\mathrm{~d}, J=8.0 \mathrm{~Hz}, 2 \mathrm{H}, \mathrm{Ph}-\mathrm{H})$, 6.83-6.86 (m, 4H, Ph-H), $4.42(\mathrm{t}, J=8.0 \mathrm{~Hz}, 1 \mathrm{H}, \mathrm{CH}), 4.01$ (s, br, $1 \mathrm{H}, \mathrm{OH}), 3.98\left(\mathrm{~s}, 3 \mathrm{H}, \mathrm{OCH}_{3}\right), 3.61\left(\mathrm{~s}, 3 \mathrm{H}, \mathrm{CO}_{2} \mathrm{CH}_{3}\right), 3.02$ $\left(\mathrm{d}, J=8.0 \mathrm{~Hz}, 2 \mathrm{H}, \mathrm{CH}_{2}\right), 2.22\left(6 \mathrm{H}, \mathrm{s}, 2 \mathrm{CH}_{3}\right) ;{ }^{13} \mathrm{C} \mathrm{NMR}(50 \mathrm{MHz}$, $\left.\mathrm{CDCl}_{3}\right) \delta: 172.2,157.5,150.3,135.7,134.9,127.9$ (2C), 127.1 (2C), 122.6, 113.4 (2C), 54.7, 51.2, 44.9, 40.5, 15.7, 15.6 (2C); GC-MS: $314\left(\mathrm{M}^{+}\right), 299,281,271,254,241 . \mathrm{C}_{19} \mathrm{H}_{22} \mathrm{O}_{4}$ requires C, 72.59; H, 7.05; found C, 72.37; H, 7.15.

4-(4-Methoxyphenyl)-6,8-dimethylchroman-2-one (29). Colorless oil. Spectroscopic data are according to the literature. ${ }^{20 a}$

Hydrolysis of compounds (19)-(25). Dihydrocoumarin 20, $21,22,23,24$ or $25(0.2 \mathrm{mmol})$ was treated with $1 \mathrm{~N} \mathrm{KOH}$ in THF $(2 \mathrm{~mL})$ at room temperature. After the work-up and chromatographic purification in a silica gel chromatographic column using dichloromethane-methanol $(8 / 2,7 / 3$ or $6 / 4$ depending on the polarity of the product), compounds 30, 31, 32, 33, 34 and 36 were isolated as pure samples.

3-(2-Hydroxy-5-(2-hydroxyethyl)phenyl)-3-(4-methoxyphenyl)propanoic acid (30). Yield 90\%; colorless oil; ${ }^{1} \mathrm{H}$ NMR $\left(\mathrm{CDCl}_{3} /\right.$ $\left.\mathrm{CD}_{3} \mathrm{OD}, 200 \mathrm{MHz}\right) \delta: 7.1$ (d, $\left.J=7.1 \mathrm{~Hz}, 2 \mathrm{H}, \mathrm{Ph}-\mathrm{H}\right), 6.61-6.84$ $(\mathrm{m}, 5 \mathrm{H}, \mathrm{Ph}-\mathrm{H}), 4.72(\mathrm{t}, J=7.5 \mathrm{~Hz}, 1 \mathrm{H}, \mathrm{CH}), 3.78\left(\mathrm{~s}, 3 \mathrm{H}, \mathrm{OCH}_{3}\right)$, $3.63\left(\mathrm{t}, J=6.9 \mathrm{~Hz}, 2 \mathrm{H}, \mathrm{CH}_{2}\right), 2.94-3.02\left(\mathrm{~m}, 2 \mathrm{H}, \mathrm{CH}_{2}\right), 2.70$ $\left(\mathrm{t}, J=6.7 \mathrm{~Hz}, 2 \mathrm{H}, \mathrm{CH}_{2}\right) ;{ }^{13} \mathrm{C} \mathrm{NMR}\left(50 \mathrm{MHz}, \mathrm{CDCl}_{3}\right) \delta: 177.6$, 159.0, 151.8, 133.1, 130.6 (2C), 129.2, 130.1, 126.2, 123.2, 117.5 (2C), 115.9, 63.0, 54.2, 42.4, 39.2, 38.5. $\mathrm{C}_{18} \mathrm{H}_{20} \mathrm{O}_{5}$ requires $\mathrm{C}$, $68.34 ; \mathrm{H}, 6.37$; found $\mathrm{C}, 68.42 ; \mathrm{H}, 6.45$.

3-(3,4-Dimethoxyphenyl)-3-(2-hydroxy-5-(2-hydroxyethyl)phenyl)propanoic acid (31). Yield 92\%; colorless oil; ${ }^{1} \mathrm{H}$ NMR $\left(\mathrm{CDCl}_{3} /\right.$ $\left.\mathrm{CD}_{3} \mathrm{OD}, 200 \mathrm{MHz}\right) \delta: 6.63-6.85(\mathrm{~m}, 6 \mathrm{H}, \mathrm{Ph}-\mathrm{H}), 4.71(\mathrm{t}, J=7.8 \mathrm{~Hz}$, $1 \mathrm{H}, \mathrm{CH}), 3.78\left(\mathrm{~s}, 3 \mathrm{H}, \mathrm{OCH}_{3}\right), 3.74\left(\mathrm{~s}, 3 \mathrm{H}, \mathrm{OCH}_{3}\right), 3.63$ $\left(\mathrm{t}, J=6.9 \mathrm{~Hz}, 2 \mathrm{H}, \mathrm{CH}_{2}\right), 2.85-3.10\left(\mathrm{~m}, 2 \mathrm{H}, \mathrm{CH}_{2}\right), 2.62$ $\left(\mathrm{t}, J=6.6 \mathrm{~Hz}, 2 \mathrm{H}, \mathrm{CH}_{2}\right) ;{ }^{13} \mathrm{C} \mathrm{NMR}\left(50 \mathrm{MHz}, \mathrm{CDCl}_{3}\right) \delta: 177.6$, $151.7,149.6$, 147.6, 130.6, 129.4, 128.5, 126.2, 123.8, 123.0, 115.9, 115.0, 112.0, 63.0, 56.0 (2C), 42.4, 39.7, 39.2. $\mathrm{C}_{19} \mathrm{H}_{22} \mathrm{O}_{6}$ requires $\mathrm{C}$, 65.88; $\mathrm{H}, 6.40$; found $\mathrm{C}, 68.48 ; \mathrm{H}, 6.32$.

3-(2-Hydroxy-5-(2-hydroxyethyl)phenyl)-3-(3,4,5-

trimethoxyphenyl)propanoic acid (32). Yield 92\%; colorless oil; ${ }^{1} \mathrm{H}$ NMR $\left(\mathrm{CDCl}_{3} / \mathrm{CD}_{3} \mathrm{OD}, 200 \mathrm{MHz}\right) \delta: 6.77-6.86(\mathrm{~m}, 2 \mathrm{H}, \mathrm{Ph}-\mathrm{H})$, $6.65(\mathrm{~d}, J=8.0 \mathrm{~Hz}, 1 \mathrm{H}, \mathrm{Ph}-\mathrm{H}), 6.45(\mathrm{~s}, 2 \mathrm{H}, \mathrm{Ph}-\mathrm{H}), 4.68$ $(\mathrm{t}, J=7.8 \mathrm{~Hz}, 1 \mathrm{H}, \mathrm{CH}), 3.70\left(\mathrm{~s}, 6 \mathrm{H}, 2 \mathrm{OCH}_{3}\right), 3.68(\mathrm{~s}, 3 \mathrm{H}$, $\left.\mathrm{OCH}_{3}\right), 3.59\left(\mathrm{t}, J=6.7 \mathrm{~Hz}, 2 \mathrm{H}, \mathrm{CH}_{2}\right), 2.81-3.07\left(\mathrm{~m}, 2 \mathrm{H}, \mathrm{CH}_{2}\right)$, $2.61\left(\mathrm{t}, J=6.7 \mathrm{~Hz}, 2 \mathrm{H}, \mathrm{CH}_{2}\right) ;{ }^{13} \mathrm{C} \mathrm{NMR}\left(50 \mathrm{MHz} \mathrm{CDCl}_{3}\right) \delta: 177.6$, $151.5,152.2$, 151.4, 140.4, 137.0, 131.4, 130.1, 126.2, 124.0, 115.9, 108.0 (2C), 63.0, 60.6, 56.1 (2C), 42.4, 40.9, 39.2. $\mathrm{C}_{20} \mathrm{H}_{24} \mathrm{O}_{7}$ requires $\mathrm{C}, 63.82 ; \mathrm{H}, 6.43$; found $\mathrm{C}, 63.72 ; \mathrm{H}, 6.38$.

3-(2-Hydroxy-5-(2-hydroxyethyl)phenyl)-3-(4-hydroxyphenyl)propanoic acid (33). Yield 88\%; colorless oil; ${ }^{1} \mathrm{H}$ NMR $\left(\mathrm{CDCl}_{3} /\right.$ $\left.\mathrm{CD}_{3} \mathrm{OD}, 200 \mathrm{MHz}\right) \delta: 7.12$ (d, $\left.J=8.7 \mathrm{~Hz}, 2 \mathrm{H}, \mathrm{Ph}-\mathrm{H}\right), 6.51-6.73$ $(\mathrm{m}, 5 \mathrm{H}, \mathrm{Ph}-\mathrm{H}), 4.65$ (t, $J=7.6 \mathrm{~Hz}, 1 \mathrm{H}, \mathrm{CH}), 3.57$ (t, $J=6.7 \mathrm{~Hz}, 2 \mathrm{H}$, $\mathrm{CH}_{2}$ ), 2.78-3.01 (m, 2H, $\left.\mathrm{CH}_{2}\right), 2.56\left(\mathrm{t}, J=6.7 \mathrm{~Hz}, 2 \mathrm{H}, \mathrm{CH}_{2}\right)$; ${ }^{13} \mathrm{C}$ NMR (50 MHz, $\mathrm{CDCl}_{3}$ ) $\delta: 177.6,154.0,151.8,139.7,130.2$ (2C), 130.1, 129.3, 126.2, 123.2, 117.9 (2C), 115.9, 63.0, 42.4, 39.2, 38.5. $\mathrm{C}_{17} \mathrm{H}_{18} \mathrm{O}_{5}$ requires C, 67.54; $\mathrm{H}, 6.00$; found $\mathrm{C}, 67.74 ; \mathrm{H}, 6.10$.

3-(4-Hydroxy-3-methoxyphenyl)-3-(2-hydroxy-5-(2-

hydroxyethyl)phenyl)propanoic acid (34). Yield 90\%; colorless oil; ${ }^{1} \mathrm{H} \mathrm{NMR}\left(\mathrm{CDCl}_{3} / \mathrm{CD}_{3} \mathrm{OD}, 200 \mathrm{MHz}\right) \delta$ : 6.59-6.86 (m, 6H, $\mathrm{Ph}-\mathrm{H}), 4.70$ (t, $J=7.5 \mathrm{~Hz}, 1 \mathrm{H}, \mathrm{CH}), 3.66\left(\mathrm{t}, J=6.6 \mathrm{~Hz}, 2 \mathrm{H}, \mathrm{CH}_{2}\right)$, 2.88-3.10 (m, 2H, $\left.\mathrm{CH}_{2}\right), 2.58\left(\mathrm{t}, J=6.6 \mathrm{~Hz}, 2 \mathrm{H}, \mathrm{CH}_{2}\right) ;{ }^{13} \mathrm{C} \mathrm{NMR}$ $\left(50 \mathrm{MHz} \mathrm{CDCl}_{3}\right) \delta: 177.6,151.7,149.2,143.6,135.1,130.1,129.5$, 128.2, 124.7, 123.0, 117.9, 115.9 (2C), 63.0, 55.9, 42.4, 39.7, 39.5. $\mathrm{C}_{18} \mathrm{H}_{20} \mathrm{O}_{6}$ requires C, 65.05; $\mathrm{H}, 6.07$; found: $\mathrm{C}, 65.25 ; \mathrm{H}, 6.12$.

3-(4-Hydroxy-3,5-dimethoxyphenyl)-3-(2-hydroxy-5-(2-hydroxyethyl)phenyl)propanoic acid (36). Yield 92\%; colorless oil; ${ }^{1} \mathrm{H}$ NMR $\left(\mathrm{CDCl}_{3} / \mathrm{CD}_{3} \mathrm{OD}, 200 \mathrm{MHz}\right) \delta: 6.58-6.95(\mathrm{~m}, 4 \mathrm{H}, \mathrm{Ph}-\mathrm{H})$, $6.43(\mathrm{~s}, 2 \mathrm{H}, \mathrm{Ph}-\mathrm{H}), 4.65(\mathrm{t}, J=7.5 \mathrm{~Hz}, 1 \mathrm{H}, \mathrm{CH}), 3.75(\mathrm{~s}, 6 \mathrm{H}$, $\left.2 \mathrm{OCH}_{3}\right), 3.66\left(\mathrm{t}, J=6.6 \mathrm{~Hz}, 2 \mathrm{H}, \mathrm{CH}_{2}\right), 2.87-2.96\left(\mathrm{~m}, 2 \mathrm{H}, \mathrm{CH}_{2}\right)$, $2.61\left(\mathrm{t}, J=6.6 \mathrm{~Hz}, 2 \mathrm{H}, \mathrm{CH}_{2}\right) ;{ }^{13} \mathrm{C} \mathrm{NMR}\left(50 \mathrm{MHz} \mathrm{CDCl}_{3}\right) \delta: 177.6$, 151.5, 148.4 (2C), 136.5, 133.8 (2C), 131.5, 130.1, 126.2, 124.0, $115.9,108.2$ (2C), 63.0, 56.1, 42.4, 40.9, 39.2. $\mathrm{C}_{19} \mathrm{H}_{22} \mathrm{O}_{7}$ requires C, 62.97; H, 6.12; found: C, 63.42; H, 6.10. 


\section{Determination of the antioxidant activity}

The antioxidant activity of tyrosol 1 and compounds 30, 31, 32, 33, 34 and 36 was determined using DPPH as a free radical in methanol. ${ }^{32}$ This ability was expressed as Efficient Concentration $\left(\mathrm{EC}_{50}=\mathrm{mmol}\right.$ of antioxidant $\left./ \mathrm{mmol} \mathrm{DPPH}\right)$ that is the concentration of antioxidant needed to decrease the initial DPPH concentration by $50 \%$. Aliquots of methanol solution containing different concentrations of the tested compound expressed as the number of mmoles of antioxidant $/ \mathrm{mmol}$ DPPH were added to $2.8 \mathrm{~mL}$ of $6 \times 10^{-5} \mathrm{M}$ methanolic DPPH solution. The decrease in absorbance was determined at $25{ }^{\circ} \mathrm{C}$ at selected $\lambda=516 \mathrm{~nm}\left(\varepsilon_{516}=10357 \pm 162 \mathrm{M}^{-1} \mathrm{~cm}^{-1}\right)$ for different ranges of time until the reaction reached a plateau. For each concentration tested, the reaction kinetics was plotted. From these graphs the percentage of remaining DPPH at the steady state was determined and corrected with respect to a control DPPH solution. The percentage of remaining DPPH values was transferred onto another graph showing the percentage of residual DPPH at the steady state as a function of molar ratio of tyrosol and cinnamic acid derivatives to DPPH. $\mathrm{EC}_{50}$ values were then extrapolated.

\section{Notes and references}

1 B. Halliwell and J. M. C. Gutteridge, Free radicals in biology and medicine, Oxford University Press, USA, 4th edn, 2007.

2 (a) A. Nunomura, G. Perry, G. Aliev, K. Hirai, A. Takeda, E. K. Balraj, P. K. Jones, H. Ghanbari, T. Wataya, S. Shimohama, S. Chiba, C. S. Atwood, R. B. Petersen and M. A. Smith, J. Neuropathol. Exp. Neurol., 2001, 60, 759; (b) J. Perry, A. Nunomora, K. Hirai, X. Zhu, M. Perez, J. Avila, R. J. Castellani, C. S. Atwood, G. Aliev, L. M. Sayre, A. Takeda and M. A. Smith, Free Radical Biol. Med., 2002, 33, 1475; (c) R. C. Seet, C. Y. Lee, E. C. Lim, J. J. Tan, A. M. Quek, W. L. Chong, W. F. Looi, S. H. Huang, H. Wang, Y. H. Chan and B. Halliwell, Free Radical Biol. Med., 2010, 48, 560.

3 (a) B. Halliwell, Biochem. J., 2007, 401, 1; (b) M. Valko, M. Izakovic, M. Mazur, C. J. Rhodes and J. Telser, Mol. Cell. Biochem., 2004, 266, 37.

4 E. S. Flamm, H. B. Demopoulos, M. L. Seligman, R. G. Poser and J. Ransohoff, Stroke, 1978, 9, 445.

5 T. M. Paravicini and R. M. Touyz, Diabetes Care, 2008, 31, S170.

6 M. F. Hill, Curr. Cardiol. Rev., 2008, 4, 259.

7 O. Firuzi, L. Fuksa, C. Spadaro, I. Bousova, V. Riccieri, A. Spadaro, R. Petrucci, G. Marrosu and L. Saso, J. Pharm. Pharmacol., 2006, 58, 951.

8 A. Mirshafiey and M. Mohsenzadegan, Immunopharmacol. Immunotoxicol., 2009, 31, 13.

9 P. M. Kris-Etherton, K. D. Hecker, A. Bonanome, S. M. Coval, A. E. Binkoski, K. F. Hilpert, A. E. Griel and T. D. Etherton, Am. J. Med., 2002, 113, 71S.

10 (a) G. Montedoro, M. Servili, M. Baldioli and E. Miniati, J. Agric. Food Chem., 1992, 40, 1571; (b) C. Manna, F. Della Ragione, V. Cucciola, A. Borriello, S. D'Angelo, P. Galletti and V. Zappia, Adv. Exp. Med. Biol., 1999, 472, 115; (c) F. Visioli, G. Bellomo and C. Galli, Biochem. Biophys. Res. Commun., 1998, 247, 60; (d) F. Visioli and C. Galli, J. Agric. Food Chem., 1998, 46, 4292.

11 (a) K. Adom, M. Sorrells and R. Liu, J. Agric. Food Chem., 2003, 51, 7825; (b) C. Gallardo, L. Jimenez and M.-T. GarciaConesa, Food Chem., 2006, 99, 455.

12 R. Di Benedetto, R. Vari, B. Scazzocchio, C. Filesi, C. Santangelo, C. Giovannini, P. Matarrese, M. D'Archivio and R. Masella, Nutr., Metab. Cardiovasc. Dis., 2007, 17, 535.

13 (a) Y.-T. Lee, M.-J. Don, P.-S. Hung, Y.-C. Shen, Y.-S. Lo, K.-W. Chang, C.-F. Chen and L.-K. Ho, Cancer Lett., 2005, 19; (b) C. W. Lee, E.-M. Son, H. S. Kim, P. Xu, T. Batmunkh, B.-J. Lee and K. A. Koo, Bioorg. Med. Chem. Lett., 2007, 17, 5462; (c) E.-Y. Ahn, Y. Jiang, Y. Zhang, E. M. Son, S. You, S.-W. Kang, J.-S. Park, J. H. Jung, B.-J. Lee and D.-K. Kim, Oncol. Rep., 2008, 19, 527; (d) I. Aissa, M. Bouaziz, F. Frikha, R. B. Mansour and Y. Gargouri, Process Biochem., 2012, 47, 2356; (e) I. Aissa, R. M. Sghair, M. Bouaziz, D. Laouini, S. Sayadi and Y. Gargouri, Lipids Health Dis., 2012, 11, 1.

14 (a) R. Bernini, E. Mincione, M. Barontini and F. Crisante, J. Agric. Food Chem., 2008, 56, 8897; (b) R. Bernini, E. Mincione, M. Barontini, F. Crisante and G. Fabrizi, Tetrahedron Lett., 2009, 50, 1307; (c) R. Bernini, F. Crisante, N. Merendino, R. Molinari, M. C. Soldatelli and F. Velotti, Eur. J. Med. Chem., 2011, 46, 439; (d) R. Bernini, F. Crisante, G. Fabrizi and P. Gentili, Curr. Org. Chem., 2012, 16, 1051; (e) R. Bernini, G. Fabrizi, L. Pouységu, D. Deffieux and S. Quideau, Curr. Org. Synth., 2012, 9, 650; $(f)$ R. Bernini, F. Crisante, M. Barontini, D. Tofani, D. V. Balducci and A. Gambacorta, J. Agric. Food Chem., 2012, 60, 7408; (g) R. Bernini, N. Merendino, A. Romani and F. Velotti, Curr. Med. Chem., 2013, 20, 655. 15 (a) J. R. Hwu, Y. S. Wein and Y. J. Leu, J. Org. Chem., 1996, 61, 1493; (b) M. A. McGuire, S. C. Shilcrat and E. Sorenson, Tetrahedron Lett., 1999, 40, 3293.

16 J. Barluenga, F. Andina and F. Aznar, Org. Lett., 2006, 8, 2703.

17 (a) V. Nair, Synth. Commun., 1987, 17, 723; (b) E. Fillion, A. M. Dumas, B. A. Kuropatwa, N. R. Malhotra and T. C. Sitler, J. Org. Chem., 2006, 71, 409.

18 T. Matsuda, M. Shigeno and M. Murakami, J. Am. Chem. Soc., 2007, 129, 12086.

19 K. Sato, T. Amakasu and S. Abe, J. Org. Chem., 1964, 29, 2971. 20 (a) J. Chenault and J.-F. E. Dupin, Heterocycles, 1983, 20, 437; (b) G. Speranza, A. Di Meo, S. Zanzola, G. Fontana and P. Manitto, Synthesis, 1997, 931; (c) G. Speranza, C. F. Morelli and P. Manitto, Synthesis, 2000, 123; (d) C. Jia, D. Piao, T. Kitamura and Y. Fujiwara, J. Org. Chem., 2000, 65, 7516; (e) A. R. Jagdale and A. Sudalai, Tetrahedron Lett., 2007, 48, 4895.

21 (a) C. E. Rodriguez-Santos and A. Echevarria, Tetrahedron Lett., 2007, 48, 4505; (b) Z. Zhang, Y. Ma and Y. Zhao, Synlett, 2008, 1091.

22 M. Aresta, J. N. Armor, M. A. Barteau, E. J. Bechman, A. T. Bell, J. E. Bercaw, C. Creutz, E. Dinjus, D. A. Dixon, 
K. Domen, D. L. Dubois, J. Eckert, E. Fujita, D. H. Gibson, W. A. Goddard, L. E. Manzaer, T. J. Marks, K. Morokuma, K. M. Nicholas, R. Periana, L. Que, J. Rostrup-Nielson, W. M. H. Sachtler, L. D. Schmidt, A. Sen, G. A. Somorjai, P. C. Stair, B. R. Stults and W. Tumas, Chem. Rev., 2001, 101, 953.

23 (a) S. Aoki, C. Amamoto, J. Oyamada and T. Kitamura, Tetrahedron, 2005, 61, 9291; (b) K. Li, L. N. Foresee and J. A. Tunge, J. Org. Chem., 2005, 70, 2881.

24 R. Bernini, E. Mincione, F. Crisante, M. Barontini, G. Fabrizi and P. Gentili, Tetrahedron Lett., 2007, 48, 7000.

25 M. Takechi, Y. Tanaka, M. Takehara, G.-I. Nonaka and I. Nishioka, Phytochemistry, 1985, 24, 2245.

26 F. Roelens, K. Huvaere, W. Dhooge, M. V. Cleemput, F. Comhaire and D. D. Keukeleire, Eur. J. Med. Chem., 2005, 40, 1042.

27 J. Sun, W.-X. Ding, X.-P. Hong, K.-Y. Zhang and Y. Zou, Chem. Nat. Compd., 2012, 48, 16.

28 T. C. Taechowisan, C. H. Lu, Y. M. Shen and S. Lumyong, Nat. Prod. Res., 2007, 21, 1104.

29 (a) C. Bailly, C. Bal, P. Barbier, S. Combes, J. P. Finet, M. P. Hildebrand, V. Peyrot and N. Wattez, J. Med. Chem., 2003, 46, 5437; (b) E. Rizzi, S. Dallavalle and L. Merlini, Synth. Commun., 2006, 36, 1117.
30 T. C. Taechowisan, C. H. Lu, Y. M. Shen and S. Lumyong, Microbiology, 2005, 151, 1691.

31 T. B. Adams, D. B. Greer, J. Doull, I. C. Munro, P. Newberne, P. S. Portoghese, R. L. Smith, B. M. Wagner, C. S. Weil, L. A. Woods and R. A. Ford, Food Chem. Toxicol., 1988, 36, 249.

32 W. Brand-Williams, M. E. Cuvelier and C. Berset, Lebensm.Wiss. Technol., 1995, 28, 25.

33 Z. Zhang, Z. Zha, C. Gan, C. Pan, Y. Zhou, Z. Wang and M.-M. Zhou, J. Org. Chem., 2006, 71, 4339.

34 A. El-Batta, C. Jiang, W. Zhao, R. Anness, A. L. Cooksy and M. Bergdahl, J. Org. Chem., 2007, 72, 5244.

35 R. P. Mahajan, S. L. Patil and R. S. Mali, Org. Prep. Proced. Int., 2005, 37, 286.

36 P. H. Kiviranta, J. Leppänen, V. M. Rinne, T. Suuronen, O. Kyrylenko, S. Kyrylenko, E. Kuusisto, A. J. Tervo, T. Järvinen, A. Salminen, A. Poso and E. A. A. Wallén, Bioorg. Med. Chem., 2007, 17, 2448.

37 F. Allais, S. Martinet and P.-H. Ducrot, Synthesis, 2009, 3571. 38 S. Saito, S. Kurakane, M. Seki, E. Takai, T. Kasai and J. Kawabata, Bioorg. Med. Chem., 2005, 13, 4191.

39 B. B. Snider and J. F. Grabowski, Tetrahedron, 2006, 62, 5171. 